natural language processing to automatically code job descriptions to SOC-2010.

Methods We recently released SOCcer 2.0. It improved on the original algorithm by 1 ) expanding the training data set to include job descriptions from population-based epidemiologic studies and 2) revising the scoring algorithm to account for nonlinearity in the classifiers. However, perfect prediction is not possible because of the lack of gold standard approach on which to train the algorithm and the brevity of the job descriptions provided by participants, which may fit multiple codes. We have adapted SOCcer to be used in the data collection process to allow the study participant to serve as their own coder when completing a web-based occupational questionnaire. SOCcer reads the participants open-ended job title and tasks responses in real time and proposes a short list of best-fitting SOC-2010 codes for each job. The study participant reviews the list and selects the code that best fits their job.

Results In a validation set of 11,943 jobs, SOCcer' highest scoring code had $50 \%$ and $63 \%$ agreement with a consensus expert-assigned code at the 6- and 3-digit level, respectively. Agreement increased linearly with algorithm score. The expert's code was in the top 3 scoring codes from SOCcer for $>70 \%$ of the jobs, lending support for providing a short list of codes for the study participants to review. Pilot testing is underway.

Conclusion Automated coding, especially in real time, has the potential to substantially reduce the efforts needed to code jobs in large epidemiologic studies and improve the codes accuracy.

\section{S-186 THE IMPACT OF JOB LOSS ON SELF-INJURY MORTALITY IN A COHORT OF AUTOWORKERS: APPLICATION OF A NOVEL CAUSAL APPROACH}

'Suzanne Dufault, Kevin Chen, Sally Picciotto, Andreas Neophytou, Ellen Eisen. 'University of California, United States

\subsection{6/OEM-2021-EPI.418}

Introduction Recent increases in national rates of suicide and fatal overdose have been linked to a deterioration of economic and social stability. The American auto industry experienced these same pressures beginning in the 1980s with the emergence of a competitive global market.

Objectives Using the United Autoworkers - General Motors cohort as a case study, we examine the impact of employment loss on these self-injury mortality events.

Methods For 29,764 autoworkers employed on or after January 1, 1970, we apply incremental propensity score (IPS) interventions, a novel causal inference approach, to examine how proportional shifts in the odds of leaving active GM employment affect the cumulative incidence of self-injury mortality.

Results Cumulative incidence of self-injury mortality was $0.87 \%$ (258 cases) at the observed odds of leaving active GM employment $(\delta=1)$ over a 45 -year period. A $10 \%$ decrease in the odds of leaving active GM employment $(\delta=0.9)$ results in an estimated $7 \%$ drop in self-injury mortality (239 cases) while a $10 \%$ increase $(\delta=1.1)$ results in a $19 \%$ increase in self-injury mortality (306 cases). We reject the null hypothesis of no incremental effect over the range of effects considered ( $\delta$ in $[0.75,1.25] ; \mathrm{P}=0.02$ ).
Conclusion These results suggest that leaving active employment increases the risk of death due to suicide or drug overdose.

\section{S-197 OCCUPATIONAL BURNOUT: DEFINITION, MEASURE AND PREDICTORS}

${ }^{1}$ Irina Guseva Canu. 'Unisanté / PMU, Switzerland

\subsection{6/OEM-2021-EPI.419}

Introduction Despite more than half a century of research on occupational burnout (OB), little is known about its prevalence, etiology, treatment, or prevention. The lack of consensus on its definition and measurement has led to the use of various arbitrary definitions and measures of $\mathrm{OB}$.

Objectives Within the Network on the Coordination and Harmonisation of European Occupational Cohorts (OMEGA$\mathrm{NET}$ ), we aimed at 1 -assessing the state of the art on $\mathrm{OB}$ recognition in the 37 OMEGA-NET-participating countries, 2elaborating a harmonized consensual definition of $\mathrm{OB}$ as a health outcome, 3-assessing the psychometric validity of the most used $\mathrm{OB}$ inventories, and 4-identifying the $\mathrm{OB}$ risk and protective factors.

Methods The first study was an on-line survey among occupational health specialists. The harmonized consensual definition resulted from the systematic review and semantic analysis of available OB definitions and a Delphi study. The two other studies were systematic reviews.

Results The survey showed that in 14 (38\%) countries OB is acknowledged as an occupational disease. The results showed a high variability in $\mathrm{OB}$ diagnosis, in assessment of its workrelatedness, and in conditions allowing compensation of patients. The harmonized definition of $\mathrm{OB}$ was formulated in accordance with the Systematized Nomenclature of Medicine Clinical Terms and consensually approved by 50 experts from 29 countries. Among five most used tools for OB measurements, only two have a moderate evidence of their psychometric validity, the CBI and OLBI. Regarding the $261 \mathrm{OB}$ predictors examined, we found a moderate evidence for the harmful effects of Job demands predictors $n=6$ ) and Negative job attitudes and for the protective effect of Adaptive coping (small effect sizes) and Leisure (small to medium effect sizes).

Conclusions These results will help harmonizing the future research, through the use of the harmonized definition and the most valid measurement tools, and facilitate decision-making and interventions regarding $\mathrm{OB}$.

\section{S-207 MULTIDIMENSIONAL EMPLOYMENT QUALITY, RETIREMENT TRAJECTORIES AND CARDIOMETABOLIC HEALTH IN LATER LIFE IN THE US}

'Sarah Andrea, Jerzy Eisenberg-Guyot, Trevor Peckham, Vanessa Oddo, Anjum Hajat. ${ }^{1}$ Rhode Island Hospital, United States and OHSU-PSU School of Public Health, United States

\subsection{6/OEM-2021-EPI.420}

Introduction The working lives of Americans have become less stable over the past several decades and older adults may be particularly vulnerable to these changes in employment quality (EQ).

Objective We examined later-life multidimensional EQ and retirement trajectories of older Americans and the potential 